\title{
Invasion Fronts and Pattern Formation in a Model of Chemotaxis in One and Two Dimensions.
}

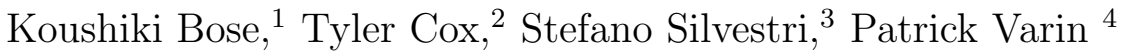

\author{
Sponsors: Matt Holzer $1^{5}$, Arnd Scheel ${ }^{6}$
}

\begin{abstract}
The purpose of this paper is to explore spatio-temporal pattern formation via invasion fronts in the one and two dimensional Keller-Segel chemotaxis model. In the one-dimensional case, simulations show that solutions that begin near an unstable equilibrium evolve into periodic patterns. These in turn evolve into new patterns through a process known as coarsening. In the two-dimensional case, we encounter only periodic patterns in the wake of the initial front. Transverse patterning only arises as a result of a transverse instability of these periodic patterns from the leading invasion front.
\end{abstract}

Keywords. chemotaxis, spreading speeds, double roots, linear stability, coarsening

\section{Introduction}

It is known that certain bacteria including E. coli and S. typhimurium respond to self-secreted chemicals through movement [2]. The process by which these bacteria move in accordance with the level of certain self-secreted chemicals is known as chemotaxis. Experiments and simulations show that chemotaxis gives rise to spatial patterns in bacterial concentrations [1,2. A standard model that is used to describe the motion of the bacteria in the presence of attracting or repelling chemicals is the Keller-Segel model [6 8]. We consider the minimal model [3], which is defined by the system of partial differential equations:

$$
\begin{aligned}
& u_{t}=\nabla \cdot(\nabla u-b u \nabla v) \\
& v_{t}=\kappa \nabla^{2} v+u-v,
\end{aligned}
$$

where $u(x, t)$ is the density of the bacteria, while $v(x, t)$ is the concentration of the chemical with $x \in \mathbb{R}^{n}$. The parameters $b>0$ denotes the chemotactic sensitivity, while $\kappa>0$ denotes the rate of diffusion of the chemical relative to the bacteria.

It is well known that the Keller-Segel model gives rise to spatial patterns in the bacterial concentration. The space-time diagram in Figure 1.1 describes the temporal progression of a solution that begins near a homogeneous equilibrium with a localized perturbation on the left boundary. The instability invades from the source of the perturbation and a periodic quasi-equilibrium appears in the wake of this unstable front. This pattern is in turn unstable and develops into another periodic pattern with twice the period via a process called coarsening. In this paper we explore the spreading speeds of these unstable fronts as well as the coarsening dynamics.

In Section 2, we consider the one-dimensional case of $x \in \mathbb{R}$. We set up the system of equations, determine the homogeneous steady states, and examine the linear stability around these points in Section 2.1. Here we see the importance of the average cell density in the stability of the system. In Section 2.2, we introduce spreading speeds and discuss how to find them using double root computations. These will be essential in determining the behavior and evolution of instabilities in the system. For a detailed discussion of front propagation into unstable states and linear spreading speeds in different systems, see [10]. In Sections 2.3 and 2.4 we find analytic expansions for the spreading speeds and selected wave numbers in the cases of small

${ }^{1}$ Department of Applied Mathematics, Brown University. Email: koushiki_bose@brown.edu

${ }^{2}$ Department of Mathematics, Georgia Institute of Technology. Email: tcox9@gatech.edu

${ }^{3}$ Department of Mathematics and Statistics, Boston University. Email: stefanos@bu.edu

${ }^{4}$ Franklin W. Olin College of Engineering. Email: patrick.varin@students.olin.edu

${ }^{5}$ Department of Mathematics, University of Minnesota. Email: mdholzer@umn.edu

${ }^{6}$ Department of Mathematics, University of Minnesota. Email: scheel@umn.edu 

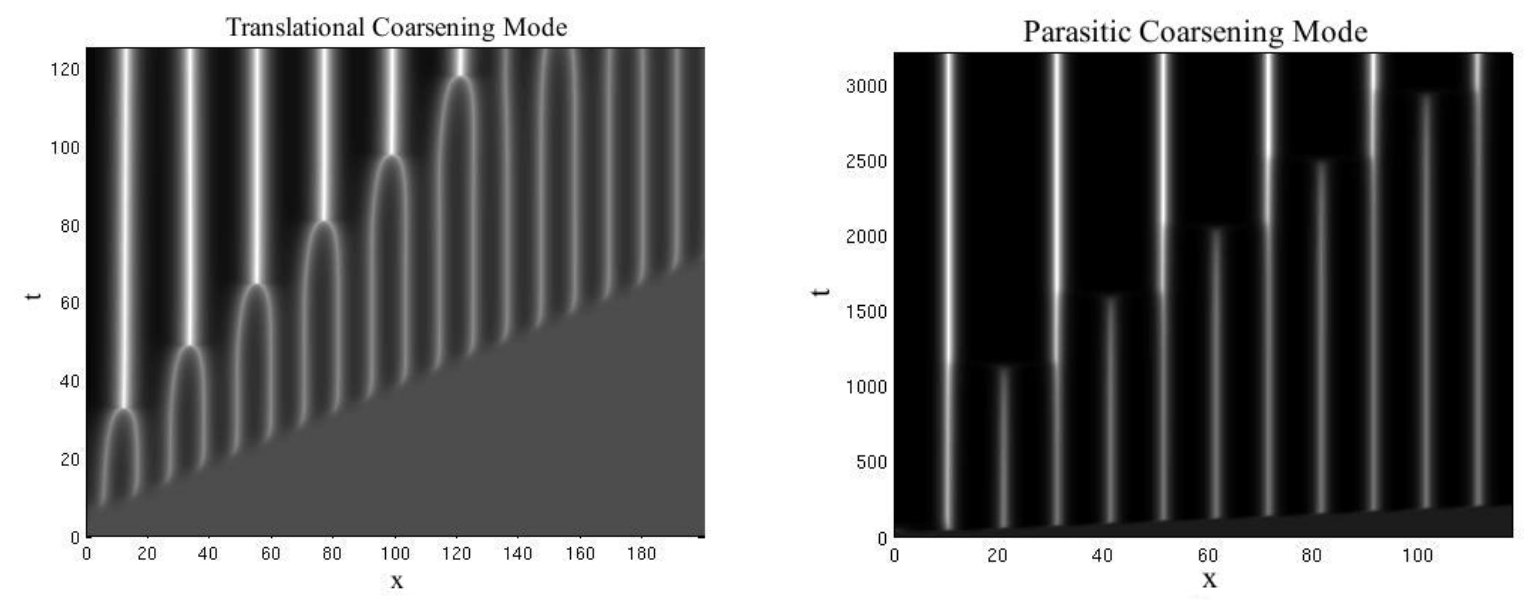

Figure 1.1: Two typical solutions to the one dimensional chemotaxis equations (1.1). Unstable homogeneous equilibria give way to periodic patterns which in turn develop into coarser patterns via a coarsening process. Here we show two different coarsening phenomena.

and large density. In Section 2.5, we study the invasion process numerically. We calculate predictions for the linear spreading speed from the complex dispersion relation and compare with direct numerical simulations. We also compare wavenumber predictions with direct simulation.

Coarsening is studied in Section 2.6. We linearize about the periodic equilibria to find unstable eigenvalues and eigenfunctions. We use numerical methods to predict two different modes of coarsening. In one of these coarsening modes, one peak simply grows at the expense of the other, while in the other, two peaks move towards each other to merge into a larger peak.

Finally in Section 3, we study the spreading in two dimensions. We allow the initial perturbation to be modulated periodically in a transverse variable and compute spreading speeds for such transversely modulated patterns. Our main results show that fronts that are not modulated in the transverse direction propagate the fastest. The primary invasion front, therefore, always admits a stripe pattern in its wake. We then study the linearization at these primary stripe patterns and observe transverse instabilities to conclude that the initial stripes will eventually evolve into another pattern with a transverse dependence.

\section{The Chemotaxis Model in One Dimension}

\subsection{Linearization and Stability Analysis}

We begin this section by considering the chemotaxis model in one dimension. Notice that the constant $b$ in Equation 1.1 may be scaled out by using the new variables $\tilde{v}=b v$ and $\tilde{u}=b u$. After performing this transformation and dropping the tilde on the variables for readability, the model becomes

$$
\begin{aligned}
& u_{t}=\left(u_{x}-u v_{x}\right)_{x}, \\
& v_{t}=\kappa v_{x x}+u-v .
\end{aligned}
$$

Next, we look at the spatially homogeneous system to determine the steady states. That is,

$$
\begin{aligned}
\frac{d}{d t} u(t) & =0, \\
\frac{d}{d t} v(t) & =u-v .
\end{aligned}
$$

The phase portrait of system 2.2 is shown in Figure 2.1 


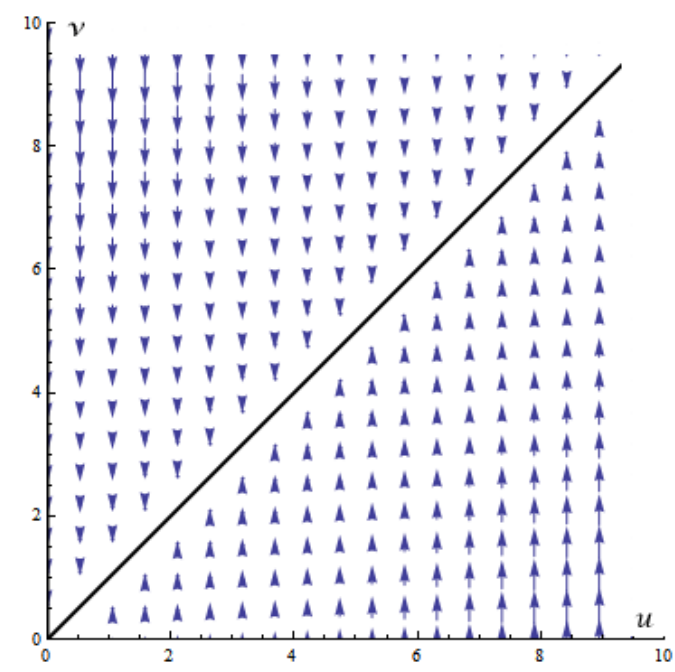

Figure 2.1: Phase portrait for the spatially homogeneous system.

As we can see, the equilibrium solutions lie on the line $u=v$. We denote the homogeneous steady states as $\left(u^{*}, v^{*}\right)$. Upon linearizing the system (2.1) near $\left(u^{*}, v^{*}\right)$, we find that

$$
\begin{aligned}
& u_{t}=u_{x x}-\chi v_{x x}, \\
& v_{t}=\kappa v_{x x}+u-v,
\end{aligned}
$$

where $\chi=u^{*}$ is the average cell density. Taking the Fourier transform, the system simplifies to $\vec{u}_{t}=A \vec{u}$, where $\vec{u}=(\hat{u}, \hat{v})^{T}$ and

$$
A=\left(\begin{array}{cc}
-k^{2} & \chi k^{2} \\
1 & -\left(\kappa k^{2}+1\right)
\end{array}\right)
$$

The eigenvalues of $A$ are given by

$$
\begin{aligned}
\lambda_{ \pm} & =\frac{1}{2} \operatorname{Tr}(A) \pm \frac{1}{2} \sqrt{(\operatorname{Tr}(A))^{2}-4 \operatorname{Det}(A)}, \text { where } \\
\operatorname{Tr}(A) & =-1-(1+\kappa) k^{2} \\
\operatorname{Det}(A) & =k^{2}\left(\kappa k^{2}+1-\chi\right) .
\end{aligned}
$$

Note that, because $\operatorname{Tr}(A)<0$, at least one eigenvalue is negative. This implies that the sign of the determinant of $A$ will determine the sign of the other eigenvalue. Recall that the system is stable if both eigenvalues are negative, and is unstable if the eigenvalues have opposite sign. It can be easily determined that $\chi=1$ is the bifurcation value separating stable states from unstable states (see Figure 2.2).

Therefore, the system contains instabilities when $\chi>1$. For non zero $k$ the dispersion relation has a maximum, which suggests that a periodic pattern will emerge from this unstable equilibrium. We are interested in the long term dynamics of the system, after a small localized perturbation of the steady state $\left(u^{*}, v^{*}\right)$. Specifically, we are interested in the speed at which the instability propagates, and the selected wavelength of the long term solution.

The value of the wavenumber $k$ that corresponds to the largest unstable eigenvalue $\lambda$ is called the most unstable mode. This gives us one possible prediction for the wavenumber selected by the linear instability. This calculation yields

$$
k^{2}=\frac{-\kappa(-1+\kappa+2 \chi)+\sqrt{\kappa \chi(1+\kappa)^{2}(-1+\kappa+\chi)}}{\kappa(-1+\kappa)^{2}}
$$



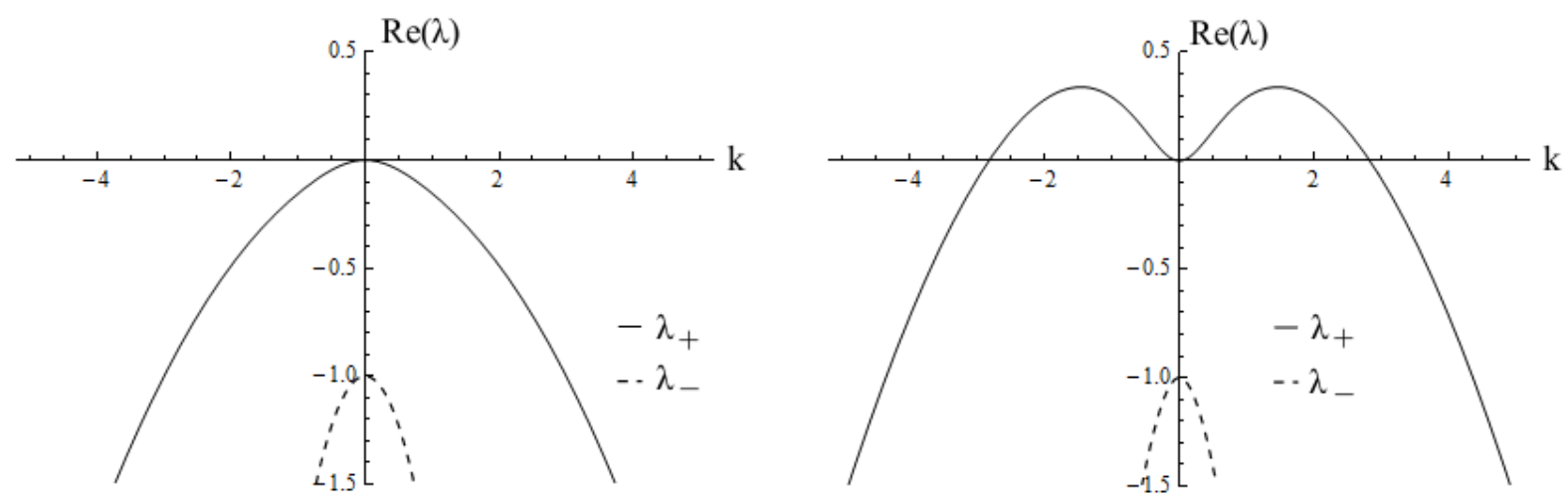

Figure 2.2: Dispersion Curves: Re $\lambda_{ \pm}$versus $k$.

In the following section, we introduce double roots as a method for finding the selected speed, wavelength and wavenumber of the instability. In Section 2.5, we will numerically calculate the selected wavenumber using double roots and compare it to the expression in 2.5).

\subsection{Spreading Speeds}

Consider the change of coordinates to a comoving frame $\xi=x-s t$, whereby the linearized system becomes

$$
\begin{aligned}
& u_{t}=u_{\xi \xi}+s u_{\xi}-\chi v_{\xi \xi}, \\
& v_{t}=\kappa v_{\xi \xi}+s v_{\xi}+u-v .
\end{aligned}
$$

Using the ansatz $\vec{u}(t, \xi)=e^{\lambda t+\nu \xi} \overrightarrow{u_{0}}$ with $\lambda, \nu \in \mathbb{C}$ yields

$$
\begin{aligned}
& \lambda u_{0}=\left(\nu^{2}+s \nu\right) u_{0}-\chi \nu^{2} v_{0}, \\
& \lambda v_{0}=\left(\kappa \nu^{2}+s \nu\right) v_{0}+u_{0}-v_{0} .
\end{aligned}
$$

Nontrivial solutions of this system relate spatial modes to temporal behaviours. These non-trivial solutions exist whenever the determinant of the above equation is zero. This determinant is known as the dispersion relation and its roots relate $\lambda$ and $\nu$,

$$
d(\lambda-s \nu, \nu)=\operatorname{det}\left(\begin{array}{cc}
\nu^{2}+s \nu-\lambda & -\chi \nu^{2} \\
1 & \kappa \nu^{2}+s \nu-1-\lambda
\end{array}\right) .
$$

Definition 1. Pinched Double Root If $d\left(\lambda^{*}-s \nu^{*}, \nu^{*}\right)=0$ and $\partial_{\nu} d\left(\lambda^{*}-s \nu^{*}, \nu^{*}\right)=0$, then $\left(\lambda^{*}, \nu^{*}\right)$ is called a double root of the dispersion relation [4].

Furthermore, a double root $\left(\lambda^{*}, \nu^{*}\right)$ is pinched if it satisfies

$$
\operatorname{Re} \nu_{ \pm}(\lambda) \rightarrow \pm \infty \text { for } \operatorname{Re} \lambda \rightarrow \infty
$$

where $\nu_{ \pm}(\lambda)$ corresponds to the roots of $d(\lambda-s \nu, \nu)=0$ which satisfy the double root condition [4].

The $\lambda^{*}$ corresponding to the pinched double root $\left(\lambda^{*}, \nu^{*}\right)$ is called the pointwise growth mode, and is a measure of the pointwise stability or instability of the system. Spatially heterogeneous patterns in chemotaxis were studied by Murray and Myerscough in [9]. They constructed a simple caricature dispersion relation to obtain estimates of the double roots.

The double roots provide information about the linear spreading speed, defined below, as well as the selected wavelength of the invasion front. Intuitively, the spreading speed measures the speed of the comoving frame for which we see a transition between growth and decay of solutions to the linear system (i.e. Re $\lambda=0$ ). 
Definition 2. Linear Spreading Speed [4] The spreading speed $s^{*}$ is defined as

$$
s^{*}=\sup \{s ; d(\lambda-s \nu, \nu) \text { possesses a pinched double root in Re } \lambda>0\} .
$$

The temporal frequency, $\omega$, associated with this speed provides information on the pattern that is produced in the wake of the front. Specifically, this invading front lays a trough or peak every $\frac{2 \pi}{\omega}$ time units. Moving back to the original stationary frame, this corresponds to the development of nucleation sites every $\frac{2 \pi s}{\omega}$ spatial units. Hence, the selected wavelength is $\frac{2 \pi s}{\omega}$.

\subsection{Analytic Spreading Speeds Using Perturbations: $\chi$ close to 1}

We begin with an investigation of linear spreading speeds near the onset of instability at $\chi=1$ using the double roots computation. Setting $\chi=1+\mu$ where $\mu \geq 0$ is small, we compute the expansions of the selected wavenumber, frequency, and speed near this unstable equilibrium.

The double root is computed according to Definition 1. The dispersion relation and its $\nu$ derivative are given by

$$
\begin{aligned}
d(\lambda-s \nu, \nu) & =(\lambda-s \nu)^{2}+\lambda\left(1+(-1-\kappa) \nu^{2}\right)+\kappa \nu^{4}+\nu^{3}(1+\kappa) s+\mu \nu^{2}-s \nu \\
\partial_{\nu} d(\lambda-s \nu, \nu) & =-2 s(\lambda-s \nu)+2 \lambda(-1-\kappa) \nu+4 \kappa \nu^{3}+3 \nu^{2}(1+\kappa) s+2 \mu \nu-s .
\end{aligned}
$$

We use the following scaling for our expansions:

$$
\begin{aligned}
\hat{\lambda} & =\lambda \kappa / \mu^{2}, \\
\hat{s} & =s \sqrt{\kappa} / \mu^{3 / 2}, \\
\hat{\nu} & =\nu \sqrt{\kappa / \mu} .
\end{aligned}
$$

We substitute these new variables into Equations 2.8 and solve for $\hat{\lambda}$ and $\hat{s}$ in terms of $\hat{\nu}$

$$
\begin{aligned}
& \hat{\lambda}=3 \hat{\nu}^{4}+\hat{\nu}^{2}+O(\mu), \\
& \hat{s}=4 \hat{\nu}^{3}+2 \hat{\nu}+O(\mu) .
\end{aligned}
$$

At leading order, the system behaves like the Cahn-Hilliard model linearized at an unstable equilibrium [4. Now we set $\hat{\nu}=\hat{\eta}+i \hat{k}$. From the definition of the spreading speed, we set $\operatorname{Re} \hat{\lambda}=0$, and to maintain physical relevance we assert that $\operatorname{Im} \hat{s}=0$. These two conditions, in addition to Equations (2.9), allow us to solve for the four variables $\hat{\eta}, \hat{k}, \hat{\lambda}$ and $\hat{s}$.

We first obtain $\hat{\eta}^{2}$ and $\hat{k}^{2}$ :

$$
\hat{\eta}^{2}=\frac{-1+\sqrt{7}}{24}+O(\mu), \quad \hat{k}^{2}=\frac{3+\sqrt{7}}{8}+O(\mu) .
$$

Recall that $\hat{\eta}=\operatorname{Re}(\hat{\nu})$ and $\hat{k}=\operatorname{Im}(\hat{\nu})$. Therefore, the above values, inserted into Equations 2.9 yield a linear spreading speed $\hat{s}$ and eigenvalue $\hat{\lambda}$. The imaginary part of the eigenvalue gives the temporal frequency $\hat{\omega}$.

$$
\hat{s}=\sqrt{\frac{2}{27}(17+7 \sqrt{7})}+O(\mu), \quad \hat{\lambda}=\frac{i(3+\sqrt{7})}{4} \sqrt{\frac{2+\sqrt{7}}{6}}+O(\mu) .
$$


Therefore, close to $\chi=1$, we find the linear spreading speed and corresponding double root

$$
\begin{aligned}
s & =\frac{\mu^{3 / 2}}{\sqrt{\kappa}} \sqrt{\frac{2}{27}(17+7 \sqrt{7})}+O\left(\mu^{5 / 2}\right), \\
\omega & =\frac{\mu^{2}}{\kappa} \frac{(3+\sqrt{7})}{4} \sqrt{\frac{2+\sqrt{7}}{6}}+O\left(\mu^{3}\right), \\
\operatorname{Re} \nu & =\sqrt{\frac{\mu}{\kappa}} \sqrt{\frac{-1+\sqrt{7}}{24}}+O\left(\mu^{3 / 2}\right), \\
\operatorname{Im} \nu & =\sqrt{\frac{\mu}{\kappa}} \sqrt{\frac{3+\sqrt{7}}{8}}+O\left(\mu^{3 / 2}\right) .
\end{aligned}
$$

These expansions give intuition for the behavior of the system close to $\chi=1$ and will be compared with simulations in Section 2.5 .

\subsection{Large $\chi$ Limit}

We now look for the spreading speed in the limit of infinite bacterial density. Let $\chi=1 / \epsilon$, where $\epsilon>0$ is small. We set $\kappa=1$ since it allows us to easily calculate explicit solutions.

We scale the variables as follows:

$$
\begin{aligned}
v & =\varepsilon \hat{v}, \\
t & =\varepsilon \hat{t}, \\
x & =\sqrt{\epsilon} \hat{x}
\end{aligned}
$$

and obtain, dropping hats,

$$
\begin{aligned}
& u_{t}=u_{x x}-v_{x x}, \\
& v_{t}=v_{x x}+u-\epsilon v .
\end{aligned}
$$

At $\epsilon=0$, we find the simplified dispersion relation

$$
d(\lambda, \nu)=\left(-\lambda+\nu^{2}\right)^{2}+\nu^{2} .
$$

It is interesting to note that this is the dispersion relation that is obtained if we omit the term $-v$ from the second equation in the system $(2.3)$. That is, considering $\chi$ large in the linear approximation is equivalent to a chemotaxis model where the chemical does not decay.

We pass into a comoving frame and obtain the dispersion relation

$$
d(\lambda-s \nu, \nu)=\left(-\lambda+s \nu+\nu^{2}\right)^{2}+\nu^{2} .
$$

From the definition of the spreading speeds we set the real part of $\lambda$ equal to 0 . This condition, in addition to the double root conditions $d=0$ and $\partial_{\nu} d=0$, allow us to solve for $s, \lambda$, and $\nu$. The imaginary part of $\lambda$ is the temporal frequency $\omega$. Finally, we rescale to obtain the original variables before the transformations in 2.11

$$
\begin{aligned}
s_{*} & = \pm \sqrt{\chi}, \\
\omega_{*} & =\chi / 2 . \\
\nu_{*} & =\frac{\mp \sqrt{\chi} \pm i \sqrt{\chi}}{2} .
\end{aligned}
$$

The linear spreading speed, frequency, and wavenumber all increase with $\chi$. The selected wavelength is $\frac{2 \pi s_{*}}{\omega_{*}}=\frac{4 \pi}{\sqrt{\chi}}$. One can verify that in this limit, the selected wavenumber coincides with the fastest linearly growing mode computed from (2.4). Figure 2.8 compares the spreading speeds obtained here with those computed numerically. This simple case gives us explicit formulas which will be useful in the two-dimensional case considered later in Section 3 . 


\subsection{Numerical Computation of Double Roots and Spreading Speed}

At this point, we have an expression for the spreading speed for the limiting values of $\chi$, i.e. $\chi \approx 1$ and $\chi \rightarrow \infty$. However, we cannot calculate the spreading speed analytically for intermediate values of $\chi$. In this regime, we will compute the spreading speed numerically as follows. The double root computation is a system of four equations, the real and imaginary part of $d(\lambda-s \nu, \nu)$ and $\partial_{\nu} d(\lambda-s \nu, \nu)$, in four variables, the real and imaginary part of $\lambda$ and $\nu$. Finding the double roots of the dispersion relation, therefore, can be done numerically using Newton's method to solve this system of equations. Verifying the pinching condition requires following the roots of the dispersion relation as $\operatorname{Re} \lambda$ becomes arbitrarily large.

Additionally, the spreading speed and the selected wavelength can be calculated from direct simulation. A hybrid of Crank-Nicolson and explicit Euler finite difference scheme was employed in the simulation of this system. The Crank-Nicolson scheme was used in the simulation of the diffusion terms, while the expicit Euler scheme was used in the simulation of nonlinear and cross-terms. The size of the finite differences $(\Delta x$ and $\Delta t$ ) varied according to the expected wavelength and the spreading speed due to restrictions in simulation time, but did not exceed values of 0.1. Simulation shows emergence of periodic patterns mentioned in Section 2.1 and the evolution of the front as it propagates into the unstable homogeneous equilibrium. Figure 2.3 shows the spacetime diagram that illustrates this phenomenon.

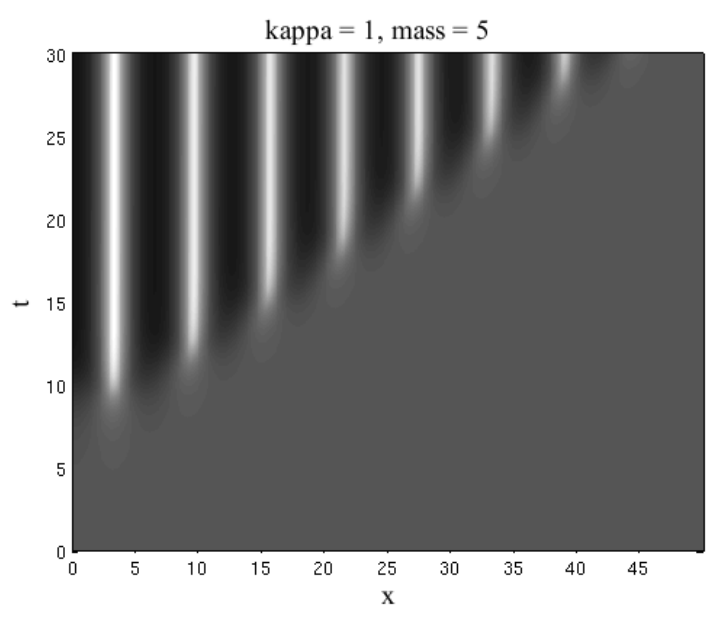

Figure 2.3: A spacetime diagram of $v$ illustrating the invasion process after an initial perturbation at the boundary. The invasion front propagates with constant speed through the unstable regime. This data is used to compare the observed spreading speed with the results of the double root computation.

Figure 2.4 compares the spreading speeds calculated via the double root computation and those calculated from simulation. The strong agreement between the spreading speeds computed from the double roots of the dispersion relation and those observed in direct simulation indicates that instabilities in the linear system, rather than the nonlinear terms, drive the propagating front and select the pattern observed in its wake. Moreover, for values of $\chi$ close to 1 , the spreading speed and wavelength also agree with the expansions obtained in Sections 2.3 (Figure 2.6.).

\subsection{Periodic Steady States and Coarsening}

Simulation indicates that the periodic pattern that emerges from an unstable homogeneous steady state is a quasi-equilibrium, which eventually develops into a different periodic equilibrium with twice the wavelength. This process is called coarsening. So far, we linearized the system about the homogeneous steady state $u^{*}=v^{*}$ to perform all our analysis in the previous sections. To investigate the coarsening dynamics we look at periodic steady state solutions. The equilibrium condition to the one dimensional chemotaxis equations 

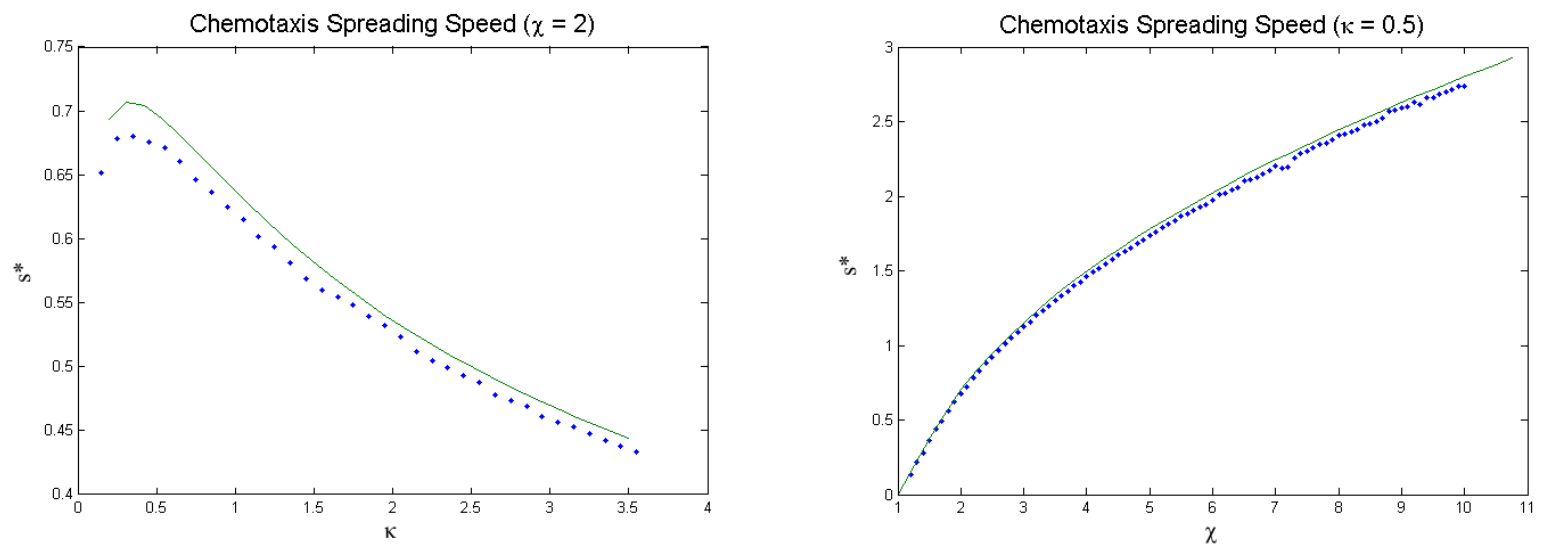

Figure 2.4: Spreading speed $s^{*}$ over two parameter ranges calculated from the double root computation $(-)$ as well as direct simulation $(\bullet)$. The strong agreement between the spreading speeds computed from the double roots of the dispersion relation and those observed in direct simulation indicates that instabilities in the linear system, rather than the nonlinear terms, drive the propagating front and select the pattern observed in its wake.
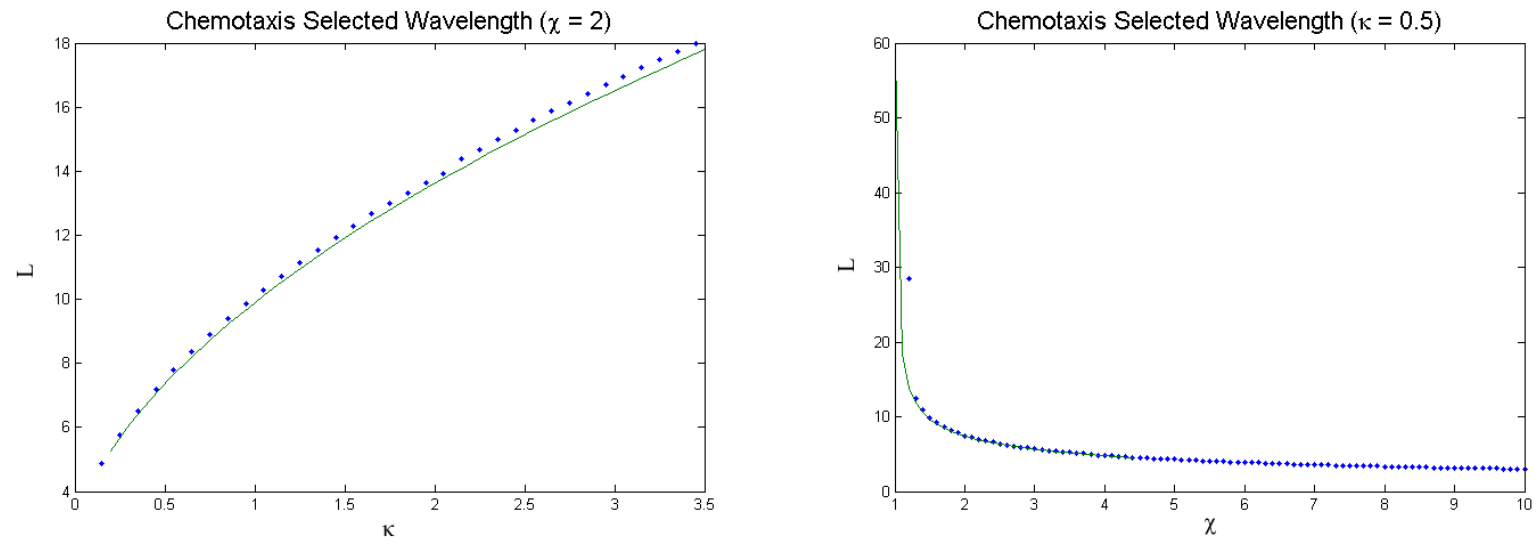

Figure 2.5: The selected wavelength $L$ over two parameter ranges calculated from the double root computation $(-)$ as well as direct simulation $(\bullet)$.

2.1 gives us

$$
\begin{aligned}
\left(u_{x}-u v_{x}\right)_{x} & =0 \\
\kappa v_{x x}+u-v & =0
\end{aligned}
$$

From the first equation in 2.14 we have

$$
\begin{aligned}
u_{x}-u v_{x} & =c \\
\Longrightarrow(\log (u)-v)_{x} & =\frac{c}{u} .
\end{aligned}
$$

Now let the period be $L$. Since we considered periodic steady state solutions, the left side vanishes when integrated over the period $L$. Because $\int_{L} \frac{1}{u}$ is necessarily positive, we find that $c=0$. Integrating once again introduces a second constant of integration, $\mu$. Simplifying we get $u=e^{\mu+v}$. Substituting into the second equation of system 2.1, we obtain

$$
\kappa v_{x x}+e^{\mu+v}-v=0
$$



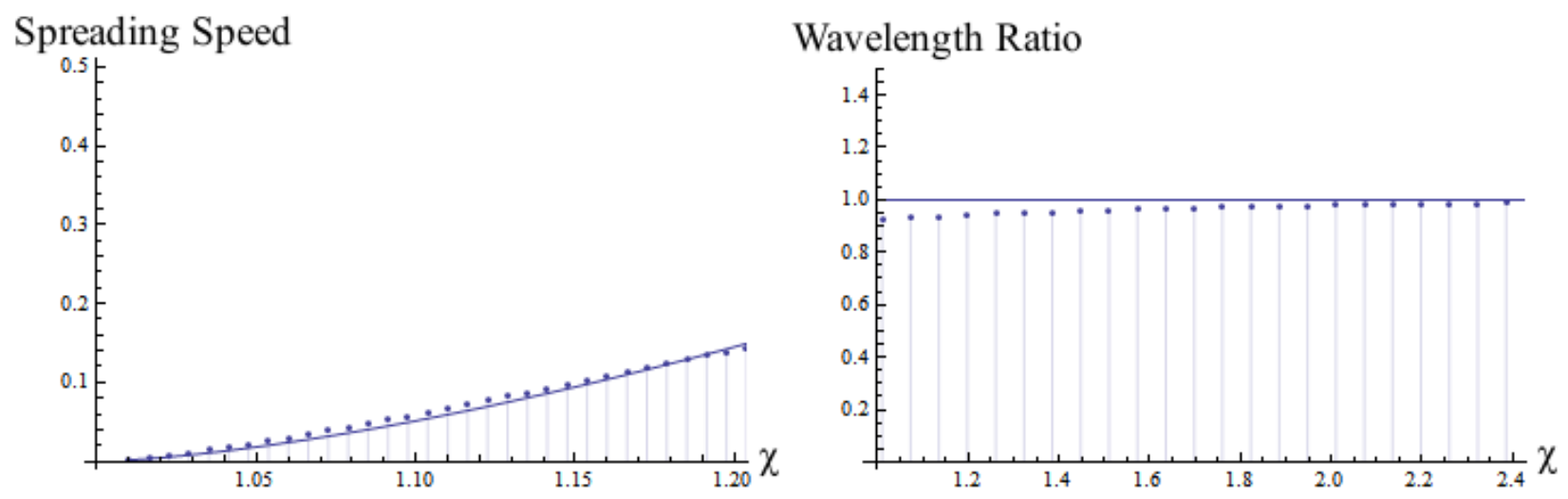

Figure 2.6: On left, comparison of spreading speed computed through expansions in 2.10 (-) and from numerics $(\bullet)$ for $\kappa=0.5$. They agree closely for $\chi$ close to 1 . On right, plot of the relative error, that is of $L$ (observed) over $L$ (predicted).

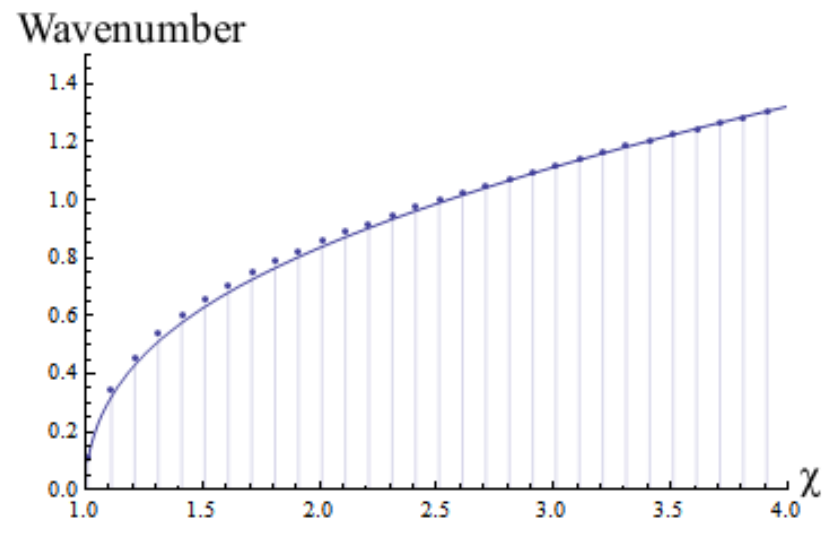

Figure 2.7: Comparison of selected wavenumber predicted $(-)$ from 2.5 and from the numerics $(\bullet)$ for $\kappa=0.5$.

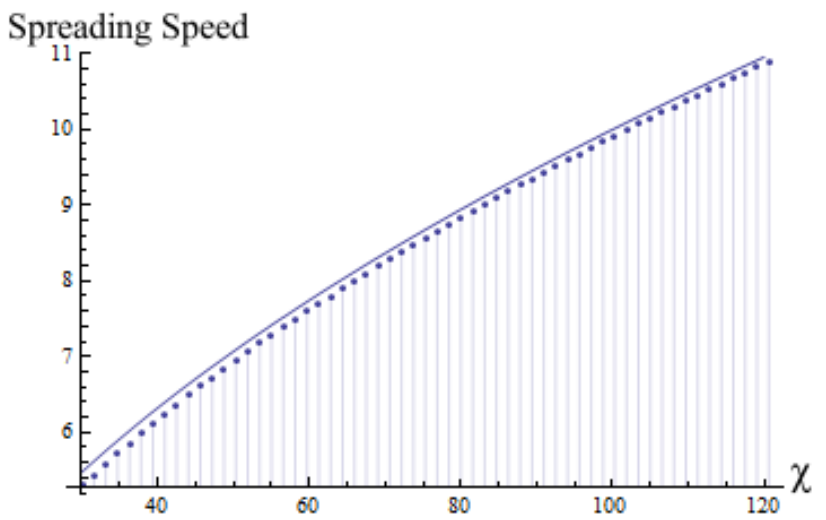

Figure 2.8: Comparison of spreading speed predicted $(-)$ from $(2.13)$ and from numerics $(\bullet)$ for $\kappa=1$. They agree more closely as $\chi$ increases, as predicted in Section 2.4

We remark that this differential equation derives form the Hamiltonian

$$
H=\kappa \frac{v_{x}^{2}}{2}-\frac{v^{2}}{2}+e^{\mu+v} .
$$

which is conservative.

The fact that this system is conservative will be of special interest when we analyze the stability of its fixed points.

It is readily observed that this system has a saddle-node bifurcation as $\mu$ is varied, specifically at $v=1$ when $\mu=-1$. Equation 2.15 has exactly one fixed point when $\mu=-1$, no fixed points when $\mu>-1$ and two fixed points when $\mu<-1$, which we shall refer to as $v^{ \pm}$. To determine the stability of these fixed points we consider the Jacobian $J$ of the the system,

$$
J=\left(\begin{array}{cc}
0 & 1 \\
\frac{1-e^{\mu+v}}{\kappa} & 0
\end{array}\right) .
$$




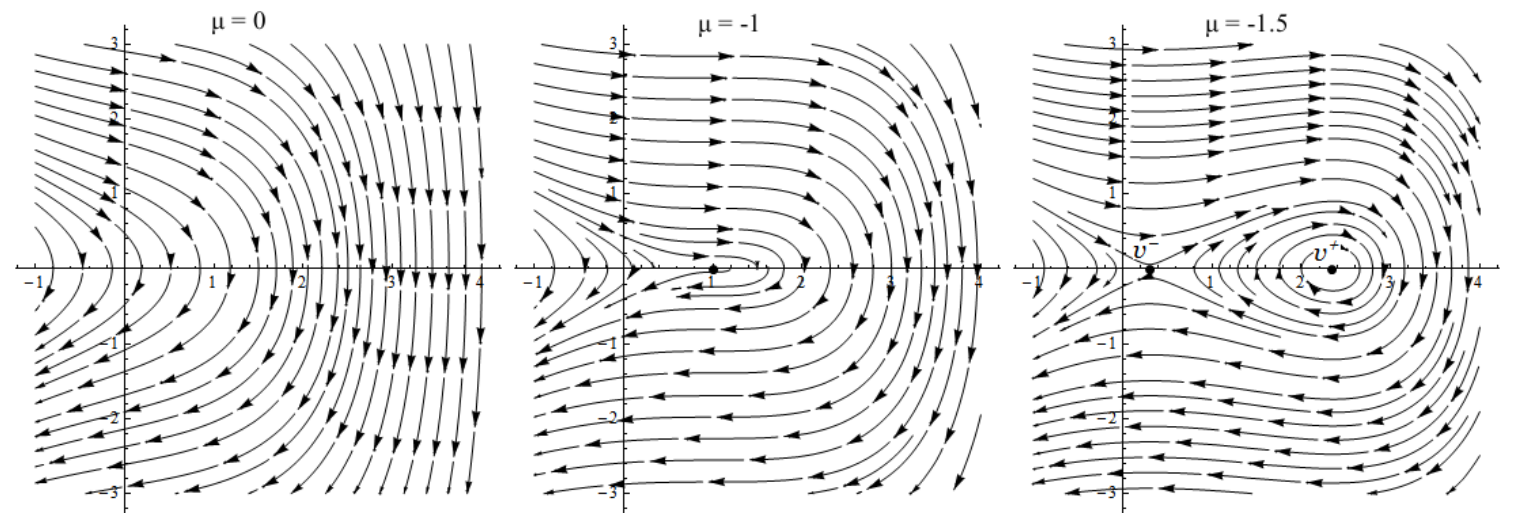

Figure 2.9: Phase portraits for the second-order ODE in Equation (2.15). Observe that a saddle-node bifurcation occurs at $\mu=-1$. When $\mu<-1$ there are no fixed points for $v$, when $\mu=-1$ there is exactly one fixed point, and when $\mu>-1$ there are two fixed points $v^{ \pm}$.

Evaluating at the fixed points the Jacobian becomes

$$
J^{ \pm}=\left(\begin{array}{cc}
0 & 1 \\
\frac{1-v^{ \pm}}{\kappa} & 0
\end{array}\right) .
$$

The trace of $J$ is always 0 , so the determinant will determine the stability of the fixed points. Specifically, because $v^{-}$and $v^{+}$emerge at $v=1$ and travel monotonically to 0 and infinity, respectively, as $\mu$ increases, one fixed point will give the Jacobian a negative determinant, corresponding to a saddle point, while the other gives the Jacobian a positive determinant corresponding to a center. Centers are usually sensitive to nonlinearities, but because this is a Hamiltonian system the stability of this center is preserved and there are periodic trajectories in the neighborhood of this fixed point.

Figure 2.9 illustrates that periodic solutions to Equation 2.15 orbit $v^{+}$and have a minimum value in $\left(v^{-}, v^{+}\right)$, thus every periodic solution to 2.15 can be uniquely identified by an initial condition $v_{x}=0$, $v \in\left(v^{-}, v^{+}\right)$, up to translations. Therefore, there is a one parameter family of periodic orbits, parameterized by their global minima. Orbits near $v^{+}$are approximated by the simple harmonic oscillator with a period of $\frac{2 \pi \kappa}{\left(1-v^{+}\right)}$. As orbits approach the homoclinic orbit that connects $v^{-}$to itself their periods grow to infinity.

\subsubsection{Linearization}

To study coarsening dynamics we first linearize the chemotaxis equations, given by system (2.1), about the periodic steady states $u^{*}, v^{*}$

$$
\begin{aligned}
& u_{t}=u_{x x}-u_{x}^{*} v_{x}-u_{x} v_{x}^{*}-u^{*} v_{x x}-u v_{x x}^{*} \\
& v_{t}=\kappa v_{x x}+u-v .
\end{aligned}
$$

or as a matrix equation $\vec{u}_{t}=A \vec{u}$ where

$$
A=\left(\begin{array}{cc}
\partial_{x x}-v_{x}^{*} \partial_{x}-v_{x x}^{*} & -u_{x}^{*} \partial_{x}-u^{*} \partial_{x x} \\
1 & \kappa \partial_{x x}-1
\end{array}\right) .
$$

For nonhomogeneous steady states, $u_{x}^{*}$ and $v_{x}^{*}$ do not vanish, so we must keep many of the terms that disappeared in 2.3). As a result the linearization is more complicated and does not allow us to analyze the system with the Fourier transform as we did before. Instead we use numerical methods to understand the system near these periodic equilibria. 


\subsubsection{Discretization of Periodic Steady States}

Because $u^{*}, v^{*}$ are characterized by a system of non-linear differential equations, 2.14), we cannot find them explicitly, rather we employ numerical techniques to approximate the linearization about these periodic equilibria. We must discretize the system to perform this computation.

Discrete solutions for $v^{*}$ were obtained by solving the initial value problem of (2.15), the corresponding $u^{*}$ solutions were obtained by the relation $u^{*}=e^{\mu+v^{*}}$. Note that because the phase plane for $v$ is reflection symmetric about $v_{x}=0$, periodic solutions are even. Thus, solving the problem over one half period is sufficient to describe solutions on the whole line. The numerical approximation to $v^{*}$ given by the solution to the initial value problem is not necessarily periodic; this is because the numerical techniques do not conserve the Hamiltonian 2.16) and therefore solutions do not lie on a closed orbit. Newton's method was used with this preliminary solution as an initial guess in order to solve the periodic boundary value problem, ensuring that the solution lies on a periodic orbit.

In the construction of the boundary value problem symmetric finite differences were used to approximate the derivative $\mathcal{D}$ with homogeneous periodic boundary conditions, while the usual second-order discrete Laplacian $\mathcal{L}$ with homogeneous periodic boundary conditions was employed

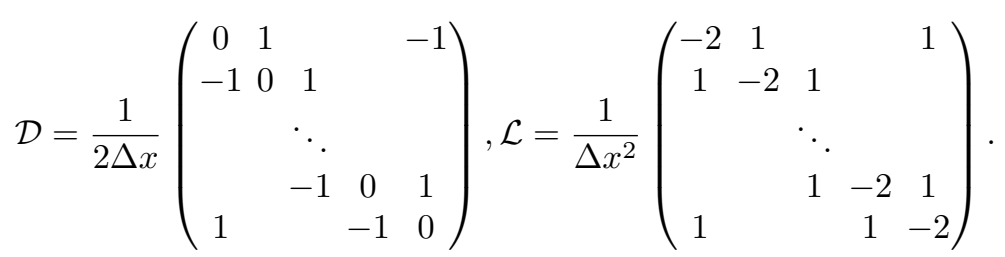

Solutions to this problem, however, lie in a two dimensional subspace-relating to translation invariance and mass conservation. To eliminate this problem, we introduced two additional equations, which limit solutions to even functions with a fixed mass $m^{*}$, and two additional variables, $\sigma$ and $\epsilon$ that artificially add mass and translate the solutions $u$ and $v$. This reduces the dimension of the solution space. The boundary value problem, then, becomes

$$
\begin{aligned}
\mathcal{L} u-\mathcal{D} u \mathcal{D} v+\sigma+\epsilon \mathcal{D} u & =0 \\
\mathcal{L} v+u-v+\epsilon \mathcal{D} v & =0 \\
u(0)-u(L) & =0 \\
u^{\prime}(0)-u^{\prime}(L) & =0 \\
\int_{0}^{L} u d x-m^{*} & =0 .
\end{aligned}
$$

The solution that results from this procedure, however, has an arbitrary wavelength, not the wavelength as would be naturally selected by a front that travels through the unstable homogeneous steady state described in Section 2.1. Thus, the numerical double-root computation described in Section 2.5 was used to determine the selected wavelength for a given $\kappa$ and average mass. With this information, Newton's method was employed iteratively to solve the boundary value problem while slowly varying the period, by changing $\Delta x$ in the $\mathcal{D}$ and $\mathcal{L}$ with a fixed number of points, in order to follow the solution to the selected wavelength. From here, solutions were followed in mass, $m^{*}$, and the corresponding selected wavelength in order to generate a family of steady state solutions for a given $\kappa$.

\subsubsection{Coarsening Modes and the Discrete Approximation to the Linearization}

The discretization described in the previous subsection allows us to rewrite the augmented matrix of equation 2.17) as:

$$
A=\left(\begin{array}{cc}
\mathcal{L}-\mathcal{D} v^{*} \mathcal{D}-\mathcal{D}^{2} v^{*}-\mathcal{D} u^{*} \mathcal{D}-u^{*} \mathcal{D}^{2} \\
I & \kappa \mathcal{L}-I
\end{array}\right) .
$$


The eigenvalues of this matrix determine the stability of these periodic steady states. When the discretized steady states $u^{*}, v^{*}$ contains only a single period, the linearization has no unstable eigenvalues and two zero eigenvalues which correspond to translation invariance and mass conservation. Using discretized steady states that contain two periods, however, allows neighboring peaks to interact and admits two distinct eigenvalues which we will refer to as $\lambda_{1 / 2}$. Figure 2.10 illustrates the dependence of these eigenvalues on the average cell density, and suggests that each eigenvalue has a regime in which it dominates.

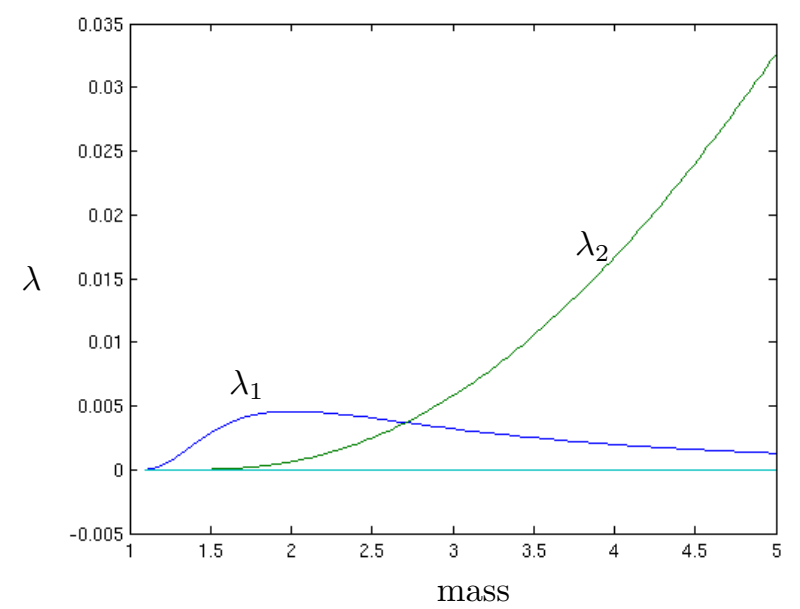

Figure 2.10: The first four eigenvalues of the linearization around a steady state with two periods. Note the two zero eigenvalues. There are two distinct positive eigenvalues corresponding to two modes of coarsening. There are clear regimes when one coarsening mode dominates over the other.

The eigenfunctions corresponding to these unstable eigenvalues give information as to the dynamics of the system near these equilibria. These eigenfunctions are plotted in Figure 2.11 and suggest two distinct mechanisms for coarsening. The eigenfunction corresponding to $\lambda_{1}$ predicts a "parasitic" coarsening process in which one spike grows while the other shrinks, the eigenfunction corresponding to $\lambda_{2}$ predicts an "aggregate" coarsening process in which the two spikes move towards each other and meet in the center of the domain.
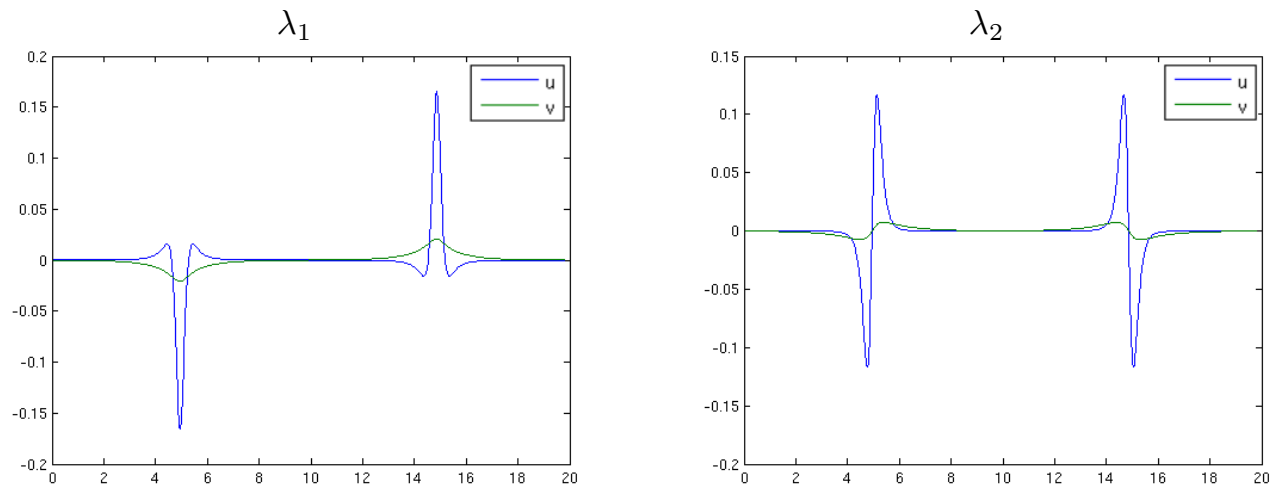

Figure 2.11: Eigenfunctions corresponding to the two unstable eigenvalues seen in Figure 2.10. These eigenfunctions describe two different coarsening modes, a "parasitic" mode $\left(\lambda_{1}\right)$ in which one peak simply grows at the expense of the other, and an "aggregate" mode $\left(\lambda_{2}\right)$ in which the two peaks move towards each other and merge to make a larger peak. 
Although these eigenfunctions cannot predict the long term behavior of the system, we predict that the parasitic eigenfunction will allow one spike to grow at the expense of the other, until the smaller spike has been completely consumed, with little to no translation. The aggregate eigenfunction, we predict, will allow spikes to move together and merge into a larger spike the center of the domain with little to no growth beforehand.

Using our numerically generated periodic steady states, we prepare a periodic pattern of two periods with a small random spatial perturbation. Direct simulation of this initial condition with periodic boundary conditions leads to coarsening. We observe that the selected coarsening mode is predicted exactly by the dominant eigenvalue, with a smooth transition from the parasitic coarsening mode to the aggregate coarsening mode in the region where $\lambda_{1} \approx \lambda_{2}$. Figure 2.12 illustrates these different coarsening modes.

$$
\lambda_{1}>\lambda_{2}
$$

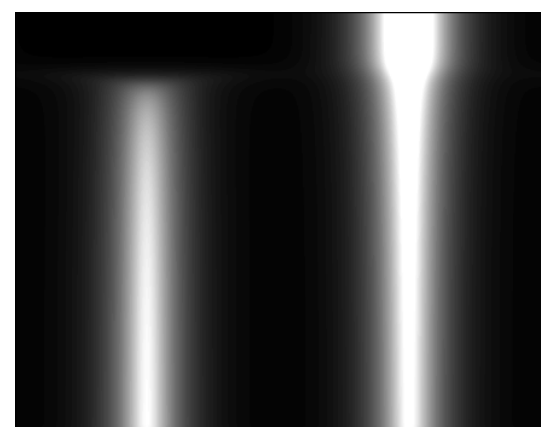

$\lambda_{1} \approx \lambda_{2}$

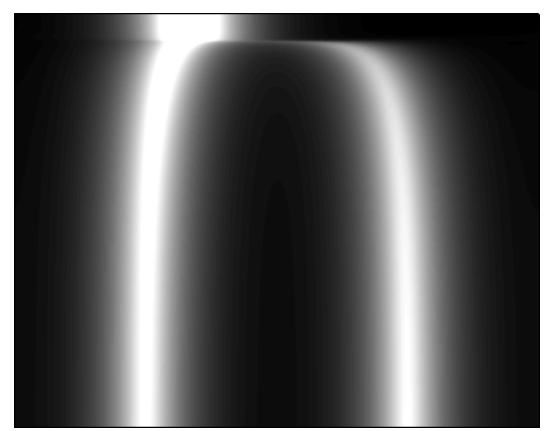

$\lambda_{1}<\lambda_{2}$

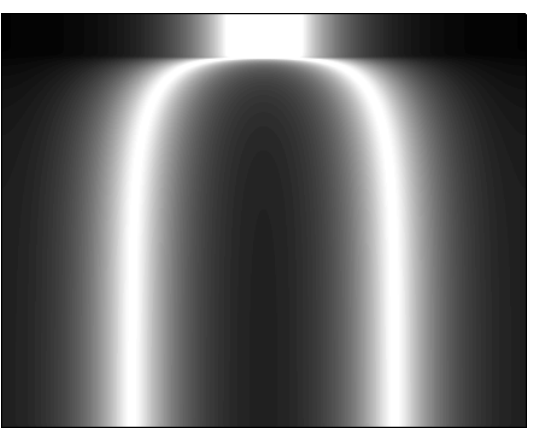

Figure 2.12: Periodic steady states perturbed with spatial white noise. Simulation results agree precisely with the dominance of the parasitic eigenfunction in the parameter regime when $\lambda_{1}>\lambda_{2}$ and the dominance of the translational eigenfunction in the parameter regime when $\lambda_{1}<\lambda_{2}$. In the intermediate regime where $\lambda_{1} \approx \lambda_{2}$ a hybrid of the two coarsening modes is observed.

In fact, this dichotomy between parasitic and aggregate coarsening appears also in the spatial invasion process; the primary invasion front is followed by a secondary coarsening front, see Figure 1.1. The transition between parasitic coarsening and translational coarsening in the spatial invasion problem, however, is not quite predicted by the eigenvalue computations here. In order to predict the dominant coarsening mode in the spatial invasion problem one must repeat the invasion speed and selected wavenumber calculations described in Section 2.2 for these selected periodic equilibria. Since the periodic equilibria are inhomogeneous, this is a much more involved process and we did not pursue that here.

\section{The Chemotaxis Model in Two Dimensions}

\subsection{Linearization and Stability Analysis}

In two space-dimensions, one can follow many of the same recipes as in the one-dimensional case. From a PDE perspective, a major difference between the one-dimensional and the two-dimensional case is the fact that solutions in bounded domains with sufficient initial mass will blow up in finite time 7 . Of course, the study of subsequent spike formation and possible coarsening is somewhat subtle, once concentrations become infinite. We will therefore introduce aggregation-limiting terms as proposed in the literature [5], replacing $u \nabla v$ by a nonlinear function $\phi(u) \nabla v$, where $\phi(u)=u$ for $u \leq u_{\max }$ and $\phi(u) \equiv u_{\max }$ otherwise. For $u_{\max }$ sufficiently large, the model then coincided with the classical Keller-Segel model 8].

In this section we are interested in whether we can study the formation of patterns in two-dimensions as an invasion problem. We work on the cylinder where $(x, y) \in \mathbb{R} \times S^{1}$, since we will only allow bounded 


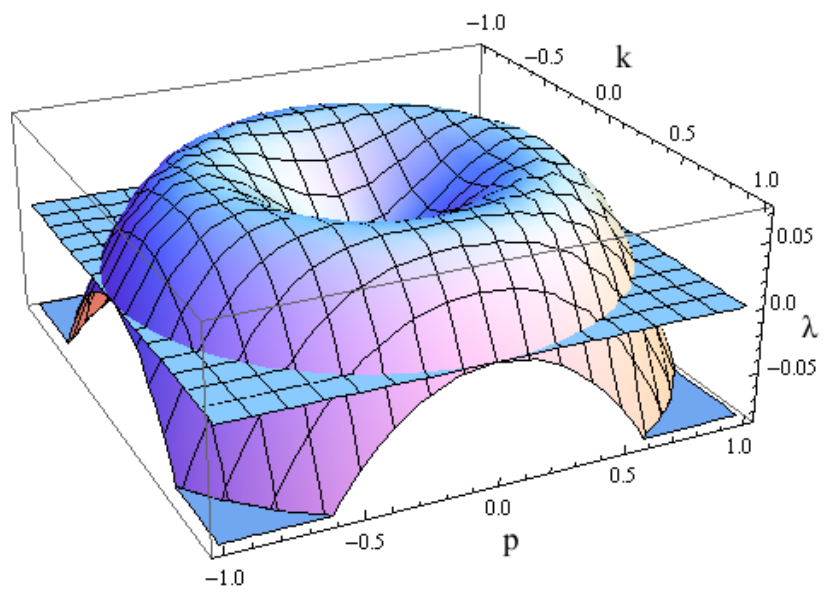

Figure 3.1: Dispersion Surface: $\lambda$ vs $k$ and $p$ for $\kappa=0.5$, and $\chi=1.5$.

modulations in the transverse direction. As in Section 2.1, the model is linearized around the homogeneous steady state $u^{*}$,

$$
\begin{aligned}
& u_{t}=u_{x x}+u_{y y}-u^{*}\left(v_{x x}+v_{y y}\right) \\
& v_{t}=\kappa\left(v_{x x}+v_{y y}\right)+u-v .
\end{aligned}
$$

We use the ansatz for the solution $\vec{u}(t, x, y)=e^{\lambda t+\nu x+i p y} \overrightarrow{u_{0}}$ where $\lambda, \nu \in \mathbb{C}$ and $p \in \mathbb{R}$. Setting $u^{*}=\chi$, the dispersion relation is

$$
d(\lambda, \nu, p)=\left(\nu^{2}-p^{2}-\lambda\right)\left(\kappa\left(\nu^{2}-p^{2}\right)-1-\lambda\right)+\chi\left(\nu^{2}-p^{2}\right) .
$$

We notice that $\chi=1$ is the bifurcation point between stable and unstable states when $p$ is small. For large $p$, we will see that the system is always stable since the growth rate is negative. Hence $\chi>1$ is a necessary but not sufficient condition for instability. We are interested in whether the parameter $p$ gives rise to instabilities and the spreading speeds associated with these. We will first consider $\chi$ close to 1 and obtain an expansion for the speading speed and selected wave number. In these expansions, the dependency of the spreading speed on $p$ tells us how transverse modulations evolve when $\chi$ is close to 1. Next, in Section 3.3, we consider the large $\chi$ limit to see how $p$ affects the growth rate of the instability. For both these cases, we conclude that for small modulations in the transverse directions, the instability spreads at a slower rate.

Similar to Figure 2.2, $\lambda$ was plotted against the transverse and the longitudinal wave numbers in Figure 3.1. This is now a surface in two-dimensions. The picture is of course isotropic, and, fixing a longitudinal wavenumber with maximal growth, we noticed that the growth rate is non-increasing as a function of the transverse wave number, all other parameters kept constant. That is, $p=0$ is the most unstable mode. When $p^{2}$ exceeds $\frac{\chi-1}{\kappa}$, the growth rate is always negative.

Finally in Section 3.4 we present a discussion of transverse instabilities that are seen when we linearize the chemotaxis system around a periodic steady state. Numerical results indicate that there are transverse instabilities that appear behind the preliminary invasion front and create patterns.

\subsection{Analytic Spreading Speed in 2D using Perturbations: $\chi$ close to 1}

To investigate the linear stability of the system around the homogeneous steady state, we need to find the double roots of the dispersion relation (Definition 1), for each fixed $p \in \mathbb{R}$. That is, we solve

$$
\begin{array}{r}
\left(\nu^{2}-p^{2}-\lambda+s \nu\right)\left(\kappa\left(\nu^{2}-p^{2}\right)-1-\lambda+s \nu\right)+\chi\left(\nu^{2}-p^{2}\right)=0 \\
(2 \nu+s)\left(\kappa\left(\nu^{2}-p^{2}\right)-1-\lambda+s \nu\right)+(2 \kappa \nu+s)\left(\nu^{2}-p^{2}-\lambda+s \nu\right)+2 \nu \chi=0 .
\end{array}
$$


Since we will consider expansions of the solutions near the unstable equilibrium $\chi=1$, we set $\chi=1+\mu$, where $\mu \geq 0$ is small. The variables are scaled as follows

$$
\begin{aligned}
\hat{\lambda} & =\lambda \kappa / \mu^{2} \\
\hat{s} & =s \sqrt{\kappa} / \mu^{3 / 2} \\
\hat{\nu} & =\nu \sqrt{\kappa / \mu} \\
\hat{p} & =p \sqrt{k / \mu} .
\end{aligned}
$$

Following the same procedure as in Section 2.3 , we find solutions

$$
\begin{aligned}
& \hat{\lambda}=3 \hat{\nu}^{4}-\hat{p}^{4}+\left(\hat{\nu}^{2}+\hat{p}^{2}\right)-2 \hat{\nu}^{2} \hat{p}^{2}+O(\mu) \\
& \hat{s}=4 \hat{\nu}\left(\hat{\nu}^{2}-\hat{p}^{2}\right)+2 \hat{\nu}+O(\mu) .
\end{aligned}
$$

Once again, $\operatorname{Re} \hat{\lambda}=0$ and $\operatorname{Im} \hat{s}=0$ for $\hat{\nu}=\hat{\eta}+i \hat{k}$. We solve for $\hat{\eta}^{2}$ and $\hat{k}^{2}$

$$
\begin{gathered}
\eta^{2}=\frac{-1+2 p^{2}+\sqrt{\left(7-4 p^{2}+4 p^{4}\right)}}{24}+O(\mu) \\
k^{2}=\frac{3-6 p^{2}+\sqrt{\left(7-4 p^{2}+4 p^{4}\right)}}{8}+O(\mu) .
\end{gathered}
$$

Now the linearized spreading speed and the double root are calculated

$$
\begin{gathered}
s=\frac{\mu^{3 / 2}}{\sqrt{\kappa}} \sqrt{\frac{2}{27}}\left(2-4 p^{2}+\sqrt{7-4 p^{2}+4 p^{4}}\right) \sqrt{-1+2 p^{2}+\sqrt{7-4 p^{2}+4 p^{4}}}+O\left(\mu^{5 / 2}\right) \\
\omega=\frac{\mu^{2}}{\kappa} \frac{3-6 p^{2}+\sqrt{7-4 p^{2}+4 p^{4}}}{4} \sqrt{\frac{2+4 p^{2}+4 p^{4}+\left(1-2 p^{2}\right) \sqrt{7-4 p^{2}+4 p^{4}}}{6}}+O\left(\mu^{3}\right) \\
\operatorname{Re} \nu=\sqrt{\frac{\mu}{\kappa} \sqrt{\frac{-1+2 p^{2}+\sqrt{\left(7-4 p^{2}+4 p^{4}\right)}}{24}}+O\left(\mu^{3 / 2}\right)} \\
\operatorname{Im} \nu=\sqrt{\frac{\mu}{\kappa}} \sqrt{\frac{3-6 p^{2}+\sqrt{\left(7-4 p^{2}+4 p^{4}\right)}}{8}}+O\left(\mu^{3 / 2}\right) .
\end{gathered}
$$

Plotting $s$ vs $p$ in Figure 3.2 we see that the fastest spreading mode is at $p=0$. Hence, for $\chi \approx 1$, the spreading speed decreases with increasing $p$ indicating that the fastest instability is the one with no transverse modulation.

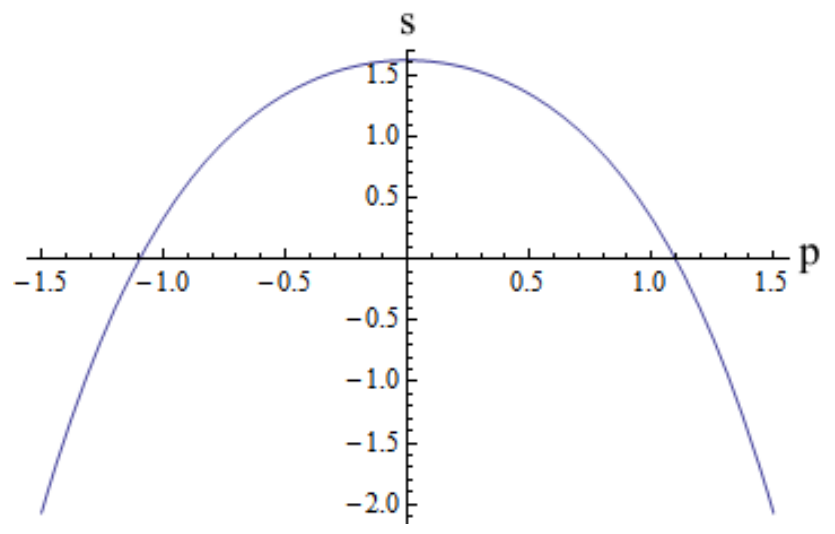

Figure 3.2: Spreading speed as a function of the transverse wave number 


\subsection{Effect of $p^{2}$ in the Large $\chi$ Limit}

Recall from Section 2.4 that setting $\chi$ large in the linear approximation is equivalent to a chemotaxis model where the chemical does not decay. Once again, $\kappa$ is taken to be 1 for simplicity, since it allows us to easily calculate explicit solutions. The dispersion relation is

$$
\left(-\lambda+s \nu+\nu^{2}-p^{2}\right)^{2}+\chi\left(\nu^{2}-p^{2}\right)=0 .
$$

When $p=0$, we know the spreading speed and selected wavenumber from Section 2.3 . We now study the spreading speed as we perturb away from $p^{2}=0$ to examine the effect of adding a small modulation in the transverse direction. Perturbation is done around the solutions $\left(\nu_{*}, \lambda_{*}\right)$ found in the one-dimensional model (2.13),

$$
\begin{aligned}
& \nu=\nu_{*}+\nu_{1} p^{2}+O\left(p^{4}\right) \\
& \lambda=\lambda_{*}+\lambda_{1} p^{2}+O\left(p^{4}\right) .
\end{aligned}
$$

Notice that Re $\lambda_{1}$ measures the rate of change of Re $\lambda$ with respect to $p^{2}$. Now from the dispersion relation we have

$$
\left(-\lambda_{*}-\lambda_{1} p^{2}+s_{*}\left(\nu_{*}+\nu_{1} p^{2}\right)+\left(\nu_{*}+\nu_{1} p^{2}\right)^{2}-p^{2}\right)^{2}+\chi\left(\left(\nu_{*}+\nu_{1} p^{2}\right)^{2}-p^{2}\right)=O\left(p^{4}\right) .
$$

From the double root computation for the one-dimensional model in Section 2.4 we have the following relations: $\left(-\lambda_{*}+s_{*} \nu+\nu_{*}^{2}\right)^{2}+\chi \nu_{*}^{2}=0$ and $\left(2 \nu_{*}+s_{*}\right)\left(-\lambda_{*}+s_{*} \nu_{*}+\nu_{*}^{2}\right)+\chi \nu_{*}=0$. Hence 3.3 simplifies and yields

$$
\lambda_{1}=-1-\frac{\chi}{2\left(-\lambda_{*}+s_{*} \nu_{*}+\nu_{*}^{2}\right)} .
$$

Recall from Section 2.4 that $s_{*}= \pm \sqrt{\chi}, \lambda_{*}=i \chi / 2, \nu_{*}=\frac{\mp \sqrt{\chi} \pm i \sqrt{\chi}}{2}$. Hence we get $\operatorname{Re} \lambda_{1}=-1 / 2$. Finally from 3.2 we have,

$$
\frac{d(\operatorname{Re} \lambda)}{d p^{2}}=-1 / 2<0
$$

Since $\lambda$ measures the growth rate of instabilities, the above result shows that adding small modulations in the transverse direction makes the instability spread at a slower rate.

\subsection{Transverse Instabilities in Periodic Patterns}

We see no evidence of transverse instabilities when we linearize about a homogeneous equilibrium; that is, there are no transverse instabilities on the leading edge of the unstable front. Thus we turn again to the linearization about $(u(x, y), v(x, y))=\left(u^{*}(x), v^{*}(x)\right)$, where $\left(u^{*}(x), v^{*}(x)\right)$ are the periodic states studied in Section 2.6. Noting that $u_{y}^{*}=v_{y}^{*}=0$, in two dimensions the linearization becomes

$$
\begin{aligned}
u(t, x, y)_{t} & =u_{x x}+u_{y y}-u_{x}^{*} v_{x}-u_{x}^{*} v_{x}-u^{*} v_{x x}-u v_{x x}^{*}-u^{*} v_{y y} \\
v(t, x, y)_{t} & =v_{x x}+v_{y y}-v+u
\end{aligned}
$$

We Fourier transform over the transverse direction to obtain the linear system $\vec{u}_{t}=\left(A-p^{2} B\right) \vec{u}$ where $\vec{u}=(u, v)^{T}$ and $\mathrm{A}$ and $\mathrm{B}$ are given by

$$
A=\left(\begin{array}{cc}
\partial_{x x}-v_{x}^{*} \partial_{x}-v_{x x}^{*}-u_{x}^{*} \partial_{x}-u^{*} \partial_{x x} \\
1 & \kappa \partial_{x x}-1
\end{array}\right), B=\left(\begin{array}{cc}
1 & -u * \\
0 & 1
\end{array}\right) .
$$

By discretizing as we did in Section 2.6.3 we can obtain the eigenvalues $\lambda$ of the matrix $\left(A-p^{2} B\right)$ and construct the dispersion relation in the transverse wavenumber $p$. This dispersion relation is plotted in Figure 3.3. The dispersion relation for the transverse wavenumber $p$ is similar to the dispersion relation illustrated in Figure 2.2 and reveals transverse instabilities that will appear behind the preliminary invasion front and create patterns in its wake. 


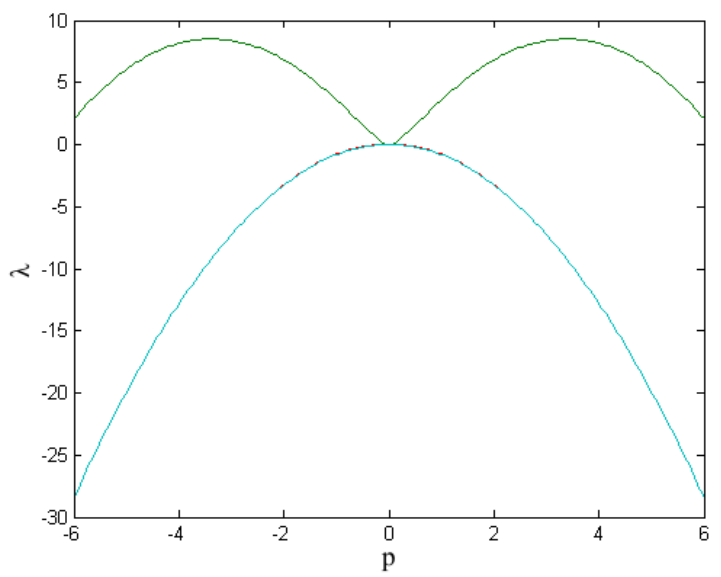

Figure 3.3: $\lambda$ as a function of $p$, the transverse wavenumber, for the chemotaxis equations linearized about a periodic equilibrium. The positive maximum in the dispersion relation reveals transverse instabilities that occur behind the preliminary invasion front.

Here we remark that when linearizing about a equilibrium that contains two periods, as pictured in Figure 3.3. the eigenvalues of $\left(A-p^{2} B\right)$ have a multiplicity of two. Moreover, the eigenvectors of these eigenvalues correspond to the parasitic coarsening modes. This may be interpreted as follows. Transverse breakup of the stripe patterns is much faster than one-dimensional coarsening (note the size of the eigenvalues in Figure 3.3 relative to those in 2.10). It is moreover largely independent of interaction between transverse stripes. As a consequence, the linear analysis does predict a transverse wavenumber and therefore a transverse spacing of a resulting periodic array of spikes. These periodic arrays may, however, shift relative to each other, forming effectively patterns of hexagons or squares, say. The linear analysis cannot distinguish between such arrangements because of the weak interaction between subsequent stripes.

\section{Conclusion}

In the one-dimensional Keller-Segel chemotaxis model, simulations showed that solutions which begin near an unstable homogeneous equilibrium evolve into periodic patterns as time increases. These patterns form a quasi-equilibrium which by a secondary invasion process develops a new pattern with double the wavelength. For cell densities close to the threshold of instability, we obtained expansions for the speed of the primary invasion front and the selected wavelength. In order to study the coarsening dynamics, we explored the linearization about periodic equilibria. The unstable eigenvalues of this linearization and their corresponding eigenfunctions revealed two different coarsening modes: a "parasitic" mode in which one peak grows at the expense of the other; and an "aggregate" mode in which neighboring peaks move towards each other and merge to form a larger peak.

In two space dimensions, we showed that the initial invasion front creates a periodic pattern with no transverse dependence. Transverse patterning arises as a result of a transverse instability of these periodic patterns in the leading edge of the front. We showed this by numerically examining the linearization at these periodic patterns.

\section{Acknowledgments}

This research was conducted during Summer 2012 in the REU: Dynamics of Pattern Formation (http://www . math.umn.edu/ scheel/reu/reu-2012.html) at the University of Minnesota Department of Mathematics, 
funded by the National Science Foundation (DMS-0806614). We would like to thank Arnd Scheel (http: //www.math.umn.edu/ scheel//) for proposing this project and for his guidance throughout the summer. We are also grateful to Matt Holzer (http://math.umn.edu/ mdholzer/) and Ryan Goh (http://math. umn.edu/ gohxx037/) for all their help and inputs throughout the project.

\section{References}

[1] E. O. Budrene And H. C. Berg, Complex patterns formed by motile cells of escherichia coli., Nature, 349 (1991).

$[2]$ - Dynamics of formation of symmetrical patterns by chemotactic bacteria, Nature, 376 (2002), pp. $49-53$.

[3] S. Childress And J. Percus, Nonlinear aspects of chemotaxis, Mathematical Biosciences, 56 (1981), pp. 217-237.

[4] R. Goh, S. Mesuro, And A. Scheel, Spatial wavenumber selection in recurrent precipitation, SIAM Journal of Applied Dynamical Systems, 10 (2011), pp. 360-402.

[5] T. Hillen and K. Painter, Global existence for a parabolic chemotaxis model with prevention of overcrowding, Advances in Applied Mathematics, 26 (2001), pp. 280 - 301.

[6] T. Hillen and K. J. Painter, A user's guide to pde models for chemotaxis, Journal of Mathematical Biology, 58 (2009), pp. 183-217.

[7] W. Jager And S. Luckhaus, On explosions of solutions to a system of partial differential equations modelling chemotaxis, Transactions of the American Mathematical Society, 329 (1992), pp. 819-824.

[8] E. Keller And L. Segel, Model for chemotaxis, Journal of Theoretical Biology, 30 (1971), pp. 225234.

[9] M. R. Myerscough and J. D. Murray, Analysis of propagating pattern in a chemotaxis system, Bulletin of Mathematical Biology, 54.

[10] W. van SaArloos, Front propagation into unstable states, Physics Reports, 386 (2003), pp. 29-222. 